\title{
LINEAR CONTROL PROBLEMS WITH TOTAL DIFFERENTIAL EQUATIONS WITHOUT CONVEXITY
}

\author{
BY
}

\author{
M. B. SURYANARAYANA
}

\begin{abstract}
Neustadt type existence theorems are given for optimal control problems described by Dieudonné-Rashevsky type total differential equations which are linear in the state variable. The multipliers from the corresponding conjugate problem are used to obtain an integral representation for the functional which in turn is used in conjunction with a Lyapunov type theorem on convexity of range of integrals to derive the existence of a usual solution from that of a generalized solution, which thus needs no convexity. Existence of optimal solutions is also proved in certain cases using an implicit function theorem along with the sufficiency of the maximum principle for optimality in the case of linear systems. Bang bang type controls are shown to exist when the system is linear in the control variable also.
\end{abstract}

Introduction. In the study of optimization problems described by linear differential systems, one feels that the existence of optimal solutions can be proved even without underlying convexity hypotheses. This vague feeling has been made precise by L. Neustadt [8] for one-dimensional problems and by the author [9c] for hyperbolic systems in the two-dimensional case. It is the purpose of this paper to establish similar existence theorems for optimal solutions for Mayer systems described by linear Dieudonné-Rashevsky type partial (total) differential equations.

Two types of theorems are presented on the existence of optimal solutions. The first type theorem is based on the criterion proposed by Cesari [2d], namely, the necessary and sufficient condition for an admissible pair to be optimal (in the case of a linear system) is that the corresponding minimum condition ("minimum principle") [2d] be satisfied a.e. in $G$, the time-domain. The existence of optimal solutions is seen in the text of this paper, to follow from the existence of a measurable function $u(x, y)$ which satisfies an auxiliary condition leading

Received by the editors August 3, 1973.

AMS (MOS) subject classifications (1970). Primary 49A20, 49A35, 93C05, 93C20, 35C15, 35F15; Secondary 49A50.

Key words and phrases. Existence without convexity, linear control problems, bang bang phenomenon, implicit function theorem, necessary conditions, multipliers. 
to the minimum condition mentioned above. The existence of the required measurable function $u(x, y)$ is proved using the implicit function theorem of E. J. McShane and R. B. Warfield [6].

The second type theorem uses the fact that the Mayer type functional can be expressed entirely in terms of the solutions of the corresponding linear conjugate problem. These representations of the functional are then used in conjunction with a Lyapunov-type theorem on integrals as in Cesari [3], to obtain a usual solution for the present optimization problem in correspondence with every generalized solution having the same value for the functional. Since generalized solutions exist without convexity, the same is therefore true for usual solutions. The bang bang phenomenon of $L$. Neustadt [8] is also observed.

Existence of solutions of the linear conjugate problem is assumed in the existence theorems for optimal solutions, and this is not restrictive in view of the previous studies (see author [9b], [9c] and J. B. Diaz-Lopez [5]). An example to illustrate this fact, is provided at the end of the paper.

2. Notations. We shall follow the basic notations of Cesari [2d]. Thus, let $G$ be a Sobolev domain in the $x y$-plane $E^{2}$; and let, for each $(x, y) \in G$, $U(x, y)$ be a closed subset of $E^{m}$. Let $\Gamma$ denote the set of all measurable functions $u(x, y)=\left(u^{1}, \cdots, u^{m}\right)$ with $u(x, y) \in U(x, y)$ a.e. in $G$. Let $W_{p}^{1}(G)$, $1 \leqslant p \leqslant \infty$, be the Sobolev class of functions $z \in L_{p}(G)$ with the generalized derivatives $z_{x}$ and $z_{y}$ also belonging to $L_{p}(G)$. Let $\Omega$ denote a class of pairs $(z, u), z(x, y)=\left(z^{1}, \cdots, z^{n}\right), u(x, y)=\left(u^{1}, \cdots, u^{m}\right)$ with $z^{i} \in W_{p}^{1}(G)$, $1 \leqslant p \leqslant \infty, i=1, \cdots, n$, and $u \in \Gamma$, satisfying differential equations and boundary conditions as follows:

We require that $[z, u]$ in $\Omega$ satisfy the $2 n$ first order partial differential equations linear in the state variable

$$
\begin{aligned}
& \partial z^{i} / \partial x=\sum_{j} A_{i j}(x, y) z^{j}+C_{i}(x, y, u), \\
& \partial z^{i} / \partial y=\sum_{j} B_{i j}(x, y) z^{j}+D_{i}(x, y, u), \quad i=1, \cdots, n,
\end{aligned}
$$

where $A_{i j}$ and $B_{i j}$ are in $L_{\infty}(G)$ and $C_{i}, D_{i}$ are in $L_{p}(G)$, for each $i=1$, $\cdots, n$ and for each $u \in \Gamma$. Further, $C_{i}$ and $D_{i}$ are assumed to be continuous in $u \in U(x, y)$, for each $(x, y) \in G$. Let $B=\partial G$ denote the boundary of $G$ and let us assume that $B$ can be divided into finitely many nonoverlapping parts $s_{h}, h=1, \cdots, N$, which will be referred to as sides of $G$. Usual conventions are assumed on the orientations of components of $B$.

Let $\sigma$ denote the arclength parameter on $B$ starting from fixed arbitrary points of $B, 0 \leqslant \sigma \leqslant L$, where $L$ is the total Jordan length of $B$. Then 
$\sigma=\sigma(x, y)$ for $(x, y) \in B$, and we shall denote by $z^{i}(\sigma)$ or $z^{i}(\sigma(x, y))$ the values of $z^{i}$ for $(x, y) \in B, i=1, \cdots, n$. We require that the pairs $(z, u)$ in $\Omega$ satisfy the boundary conditions:

(I) For each $h=1, \cdots, N$ and for each $i$ of a collection $\{i\}_{h}$ of indices $i=1, \cdots, n$ depending on $h$ (which may be empty), let

$$
z^{i}(\sigma)=\varphi_{h i}(\sigma) \text { for } \sigma \in s_{h} \text { (a.e.). }
$$

Here, $\varphi_{h i}(\sigma)$ are functions on $s_{h}$ of class $L_{p}\left(s_{h}\right)$.

We shall consider the problem of minimum in $\Omega$ of the functional

$$
I[z, u]=\sum_{h=1}^{N} \sum_{i=1}^{n} \int_{s_{h}}\left[P_{h i}(\sigma) z^{i}(\sigma) d x+Q_{h i}(\sigma) z^{i}(\sigma) d y\right],
$$

where $P_{h i}(\sigma), Q_{h i}(\sigma)$ are given functions $P_{h i}, Q_{h i} \in L_{q}\left(s_{h}\right)$ for some $q \geqslant 1$, $1 / p+1 / q \leqslant 1, h=1, \cdots, N, i=1, \cdots, n$.

Let $t=t(\sigma)$ denote the tangent vector along the side $s_{h}$ and let $\cos t x$, $\cos t y$ denote the direction cosines of $t$. Then, $d x=(\cos t x) d \sigma$ and $d y=$ $(\cos t y) d \sigma$ so that if $\pi_{h i}(\sigma)=P_{h i}(\sigma) \cos (t x)+Q_{h i}(\sigma) \cos (t y), i=1, \cdots, n$, then

$$
I[z, u]=\sum_{h=1}^{N} \sum_{i=1}^{n} \int_{s_{h}} \pi_{h i}(\sigma) z^{i}(\sigma) d \sigma
$$

If a side $s_{h}$ of $G$ is a segment parallel to the $y$-axis (resp. $x$-axis) then $P_{h i}$ (resp. $Q_{h i}$ ) can be given arbitrary values. We may assume them to be identically zero. Boundary conditions (I) and the expression (2.2) for the functional do not interfere, that is, we assume that, for every pair $h, i$ with $i \in\{i\}_{h}$, the corresponding functions $P_{h i}, Q_{h i}$ are identically zero.

As usual, we say that a pair $(z, u)$ in $\Omega$ is optimal for the functional (2.2) if $I[z, u] \leqslant I[\tilde{z}, \tilde{u}]$ for every pair $(\tilde{z}, \tilde{u})$ in $\Omega$.

3. The Hamiltonian. If $\lambda=\left(\lambda_{1}, \cdots, \lambda_{n}\right), \mu=\left(\mu_{1}, \cdots, \mu_{n}\right)$ denote any two real vector variables and $(x, y) \in G, z=\left(z^{1}, \cdots, z^{n}\right) \in E^{n}, u=$ $\left(u^{1}, \cdots, u^{m}\right) \in U(x, y)$, we define $M=\{(x, y, u) \mid(x, y) \in G, u \in U(x, y)\}$ and the Hamiltonian $H$ as the real-valued function defined on $M \times E^{3 n}$,

$$
\begin{aligned}
H(x, y, u, z, \lambda, \mu)= & \sum_{j=1}^{n}\left[\lambda_{j} f_{j}(x, y, u, z)+\mu_{j} g_{j}(x, y, u, z)\right] \\
= & \sum_{j=1}^{n} \sum_{i=1}^{n}\left[\lambda_{j} A_{j i} z^{i}+\mu_{j} B_{j i} z^{i}\right] \\
& +\sum_{j=1}^{n}\left[\lambda_{j} C_{j}(x, y, u)+\mu_{j} D_{j}(x, y, u)\right]
\end{aligned}
$$


4. The linear conjugate problem. We shall denote by $\lambda(x, y)=\left(\lambda_{1}, \cdots, \lambda_{n}\right)$, $\mu(x, y)=\left(\mu_{1}, \cdots, \mu_{n}\right)$ any pair of functions defined in $G, \lambda_{i}, \mu_{i} \in W_{p}^{1}(G)$, $i=1, \cdots, n$; we shall denote by $\Lambda_{h i}(\sigma)$ the expression

$$
\Lambda_{h i}(\sigma)=-\mu_{i}(\sigma) \cos t x+\lambda_{i}(\sigma) \cos t y, \quad \sigma \in s_{h},
$$

where $i=1, \cdots, n, h=1, \cdots, N$. We shall assume that $\lambda_{i}, \mu_{i}, i=1, \cdots$, $n$, satisfy the system of $n$ first order partial differential equations

$$
\begin{aligned}
\frac{\partial \lambda_{i}}{\partial x}+\frac{\partial \mu_{i}}{\partial y}=-\frac{\partial H}{\partial z^{i}} \equiv \sum_{j=1}^{n}\left(\lambda_{j} A_{j i}+\mu_{j} B_{j i}\right) \\
\quad i=1, \cdots, n,(x, y) \in G, \text { a.e., }
\end{aligned}
$$

with boundary conditions

$$
\Lambda_{h i}(\sigma)=\pi_{h i}(\sigma), \quad \sigma \in s_{h}, \text { a.e., }
$$

for every $h=1, \cdots, N$, and for each $i \notin\{i\}_{h}$. Note that the values of $\Lambda_{h i}$ are not assigned on $s_{h}$ for $i \in\{i\}_{h}$. If a side $s_{h}$ is a segment parallel to the $x$ axis then $\cos t y=0 ; \cos t x= \pm 1$ so that (4.3) reduces to ${ }^{\circ}-\mu_{i}(\sigma)=P_{h i}(\sigma)$, $\sigma \in s_{h}, i \notin\{i\}_{h}$. Similarly, if a side $s_{h}$ is a segment parallel to the $y$-axis, then $\cos t x=0, \cos t y= \pm 1$ and (4.3) becomes $\lambda_{i}(\sigma)=Q_{h i}(\sigma), \sigma \in s_{h}, i \notin\{i\}_{h}$.

Relations (4.2) and (4.3) represent the form of the conjugate problem for the problem of minimum under consideration as determined by Cesari [2d], with the remark that, in the present situation, the solutions $\left(\lambda_{i}, \mu_{i}\right), i=1, \cdots, n$, if they exist, are independent of the control variable $u$, and the state variable $z$. We make the following general assumptions:

(II) The linear system of partial differential equations (4.2), (4.3) has some solution $(\lambda, \mu)$ with $\lambda_{i}, \mu_{i} \in W_{q}^{1}(G), i=1, \cdots, n, 1 / p+1 / q \leqslant 1$.

(III) For every choice of the function $u(x, y)=\left(u^{1}, \cdots, u^{m}\right), u \in \Gamma$, the system (2.1), with boundary conditions (I) has some solution $z(x, y)=$ $\left(z^{1}, \cdots, z^{n}\right)$, with $z^{i} \in W_{p}^{1}(G), i=1, \cdots, n$, and $(z, u) \in \Omega$.

5. Integral representation. If $(z, u)$ is a pair in $\Omega$, if $(\lambda, \mu)$ is a solution of (4.2) and (4.3), the corresponding conjugate problem, then,

$$
\begin{aligned}
I[z, u] & =\sum_{h=1}^{N} \sum_{i=1}^{n} \int_{s_{h}} \pi_{h i}(\sigma) z^{i}(\sigma) d \sigma \\
& =\sum_{h=1}^{N} \sum_{i \notin\{i\}_{h}} \int_{s_{h}} \pi_{h i}(\sigma) z^{i}(\sigma) d \sigma=\sum_{h=1}^{N} \sum_{i \notin\{i\}_{h}} \int_{s_{h}} \Lambda_{h i}(\sigma) z^{i}(\sigma) d \sigma
\end{aligned}
$$

since $\pi_{h i}(\sigma)=0$ for $\sigma \in s_{h}, i \in\{i\}_{h}$ and $\pi_{h i}(\sigma)=\Lambda_{h i}(\sigma)$ for $\sigma \in s_{h}, i \notin$ $\{i\}_{h}$. Then, 


$$
\begin{aligned}
I[z, u]= & \sum_{h=1}^{N} \sum_{i=1}^{n} \int_{s_{h}} \Lambda_{h i}(\sigma) z^{i}(\sigma) d \sigma-\sum_{h=1}^{N} \sum_{i \in\{i\}_{h}} \int_{s_{h}} \Lambda_{h i}(\sigma) z^{i}(\sigma) d \sigma \\
= & \sum_{i=1}^{n} \sum_{h=1}^{N} \int_{s_{h}}\left[-\mu_{i}(\sigma) z^{i}(\sigma) d x+\lambda_{i}(\sigma) z^{i}(\sigma) d y\right] \\
& -\sum_{h=1}^{N} \sum_{i \in\{i\}_{h}} \int_{s_{h}} \Lambda_{h i}(\sigma) \varphi_{h i}(\sigma) d \sigma
\end{aligned}
$$

and by Green's theorem,

$$
I=\nu+\sum_{i=1}^{n} \int_{G}\left[\left(\lambda_{i} z_{x}^{i}+\mu_{i} z_{y}^{i}\right)+\left(\lambda_{i x}+\mu_{i y}\right) z^{i}\right](x, y) d x d y
$$

where

$$
v=\sum_{h=1}^{N} \sum_{i \in\{i\}_{h}} \int_{s_{h}} \Lambda_{h i}(\sigma) \varphi_{h i}(\sigma) d \sigma
$$

In view of the conditions (2.1), (4.2), (4.3) the above equation becomes

$$
\begin{aligned}
I=\nu & +\sum_{i=1}^{n} \int_{G}\left[\sum_{j=1}^{n}\left(\lambda_{i} A_{i j} z^{j}+\mu_{i} B_{i j} z^{j}\right)+\lambda_{i} C_{i}+\mu_{i} D_{i}-\left(\frac{\partial H}{\partial z^{i}}\right) z^{i}\right] \\
=\nu & +\sum_{i=1}^{n} \sum_{j=1}^{n} \int_{G}\left(\lambda_{i} A_{i j} z^{j}+\mu_{i} B_{i j} z^{j}\right)+\sum_{i=1}^{n} \int_{G}\left(\lambda_{i} C_{i}+\mu_{i} D_{i}\right) \\
& -\sum_{i=1}^{n} \sum_{j=1}^{n} \int_{G}\left(\lambda_{i} A_{j i} z^{i}+\mu_{j} B_{j i} z^{i}\right)
\end{aligned}
$$

so that

$$
\begin{aligned}
I[z, u]=\nu+\sum_{i=1}^{n} \int_{G}\left[\lambda_{i}(x, y) C_{i}(x, y, u(x, y))\right. \\
\\
\left.+\mu_{i}(x, y) D_{i}(x, y, u(x, y))\right] d x d y .
\end{aligned}
$$

REMARK 2. The integral representation (5.3) of the functional will be used in $\S 8$ below.

REMARK 3. It is to be observed that if $\left(z_{1}, u_{1}\right)$ and $\left(z_{2}, u_{2}\right)$ denote any two pairs in $\Omega$ and if $(\lambda, \mu)$ is the solution of (4.2), (4.3) (independent of $(z, u)$ ), then the above argument can be applied to $z_{1}-z_{2}=z$, and the following increment formula is obtained for $\Delta I=I\left[z_{1}, u_{1}\right]-I\left[z_{2}, u_{2}\right]$. 


$$
\begin{gathered}
\Delta I=\int_{G}\left[H\left(x, y, z_{1}, u_{1}, \lambda, \mu\right)-H\left(x, y, z_{1}, u_{2}, \lambda, \mu\right)\right] d x d y \\
=\int_{G} \sum_{i=1}^{n}\left[\lambda_{i}(x, y)\left(C_{i}\left(x, y, u_{1}\right)-C_{i}\left(x, y, u_{2}\right)\right)\right. \\
\left.\quad+\mu_{i}(x, y)\left(D_{i}\left(x, y, u_{1}\right)-D_{i}\left(x, y, u_{2}\right)\right)\right] .
\end{gathered}
$$

6. Minimum condition. A pair $(z, u) \in \Omega$ together with a solution $(\lambda, \mu)$ (with $\lambda_{i}, \mu_{i} \in W_{q}^{1}(G), i=1, \cdots, n$ ) of the corresponding conjugate problem (4.2), (4.3) is said to satisfy the minimum property provided

$$
\begin{aligned}
& H(x, y, z(x, y), u(x, y), \lambda(x, y), \mu(x, y)) \\
& \leqslant H(x, y, z(x, y), u, \lambda(x, y), \mu(x, y))
\end{aligned}
$$

for all $u \in U(x, y)$ and almost all $(x, y) \in G$.

In the present case, the inequality above is guaranteed by the following auxiliary condition:

$$
\begin{array}{r}
\sum_{j=1}^{n}\left[\lambda_{j}(x, y) C_{j}(x, y, u(x, y))+\mu_{j}(x, y) D_{j}(x, y, u(x, y))\right] \\
\leqslant \sum_{j=1}^{n}\left[\lambda_{j}(x, y) C_{j}(x, y, u)+\mu_{j}(x, y) D_{j}(x, y, u)\right]
\end{array}
$$

for all $u \in U(x, y)$ and almost all $(x, y) \in G$.

THEOREM 1. If $(z, u) \in \Omega$ and $(\lambda, \mu)$ is a solution of (4.2), (4.3) then $(z, u)$ is optimal if $(z, u, \lambda, \mu)$ satisfies the minimum condition (6.1).

Proof. The condition (6.2) (which is equivalent to (6.1)) implies that the integrand in (5.4) is $\geqslant 0$ for almost all $(x, y) \in G$. Hence $\Delta I \geqslant 0$, that is, $I[z, u] \leqslant I \tilde{z}, \tilde{u}]$.

REMARK 4. The above condition (6.1) is also necessary for optimality if $U(x, y)=U$ is a fixed compact subset of $E^{m}$ (see Cesari [2d]).

Theorem 1 above can be used to prove the existence of optimal solutions, by showing that there exist control functions satisfying the minimum condition (6.2). We need the following statement of the implicit function theorem (see McShane and Warfield [6] and Cesari [3]).

Proposition 1. Given any two metric spaces $R$ and $S$ of which $R$ is a countable union of compact subspaces, and given a measure space $G$, let $F: R \rightarrow S$ be any continuous function and let $\theta: G \rightarrow S$ be any measurable function with $\theta(G) \subset F(R)$. Then there exists a measurable map $\eta: G \rightarrow R$ such that $\theta=F \eta$ that is $\theta(x)=F(\eta(x))$, all $x \in G$.

THEOREM 2. Let $G$ be compact and let $U(x, y)$ be a compact subset of 
$E^{m}$ for each $(x, y) \in G$ and let $M=\{(x, y, u) \mid u \in U(x, y),(x, y) \in G\}$ be closed. Let assumptions (II) and (III) hold. Let

$$
F(x, y, u)=\sum_{j=1}^{n} \lambda_{j}(x, y) C_{j}(x, y, u)+\mu_{j}(x, y) D_{j}(x, y, u)
$$

be continuous on $M$. Then there is an optimal pair $(z, u) \in \Omega$.

Proof. Let $i(x, y)=\min [F(x, y, u) \mid u \in U(x, y)]$. Then $i(x, y)$ is defined and measurable on $G$. Applying the implicit function theorem to the pair of functions $F: M \rightarrow E^{1}$ and $i: G \rightarrow E^{1}$, it follows that there is a measurable function $u$ on $G$ with $u(x, y) \in U(x, y)$ and $F(x, y, u(x, y))=i(x, y) \leqslant$ $F(x, y, u)$ for all $u \in U(x, y)$, for almost all $(x, y) \in G$. Thus, $u$ satisfies (6.2). Let $z \in\left(W_{p}^{1}(G)\right)^{n}$ guaranteed by (III) corresponding to this $u$, with $(z, u) \in \Omega$. This $(z, u)$ is then, by Theorem 1, the required optimal pair.

REMARK 5. Even though Theorem 2 shows the existence of optimal solutions without convexity requirement, the hypotheses of compactness of $U(x, y)$ and continuity of $F(x, y, u)$ are rather stringent. These hypotheses are considerably relaxed by following a different approach shown below.

7. Generalized solutions. Let us assume the notations of $\S 2$ and consider the differential system

$$
\begin{aligned}
& z_{x}^{i}=\sum_{j} A_{i j} z^{j}+C_{i}(x, y, u) \\
& z_{y}^{i}=\sum_{j} B_{i j} z^{j}+D_{i}(x, y, u),
\end{aligned}
$$

with boundary data

$$
z^{i}(\sigma)=\varphi_{h i}(\sigma), \quad \sigma \in s_{h}, \quad i \in\{i\}_{h} .
$$

We assume control constraints of the form

$$
u(x, y) \in U(x, y) \text { a.e. in } G
$$

where $U(x, y)$ is a closed subset of $E^{m}$ for each $(x, y) \in G$. A pair of functions $(z, u), z \in\left(W_{p}^{1}(G)\right)^{n}, u \in \Gamma$, satisfying (7.1)-(7.3) is referred to as a "usual solution" of the differential system.

Let $\rho(x, y)=\left(\rho^{1}, \cdots, \rho^{\eta}\right)$ be a measurable function defined on $G$ such that $\rho^{i}(x, y) \geqslant 0, i=1, \cdots, r, \rho^{1}+\cdots+\rho^{r}=1,(x, y) \in G$. Let $u_{1}$, $\cdots, u_{r}$ be measurable on $G$, with values at $(x, y)$ in $U(x, y)$. Then, the pair $(z, v)$ with $v=\left(\rho, u_{1}, \cdots, u_{r}\right)$ and $z(x, y)=\left(z^{1}, \cdots, z^{n}\right)$ is said to be a generalized solution of the differential system (7.1), (7.2) if 


$$
\begin{aligned}
& z_{x}^{i}=f_{1}(x, y, z, v) \equiv \sum_{j=1}^{n} A_{i j} z^{j}+\sum_{j=1}^{r} \rho^{j} C_{i}\left(x, y, u_{j}\right), \\
& z_{y}^{i}=f_{2}(x, y, z, v) \equiv \sum_{j=1}^{n} B_{i j} z^{j}+\sum_{j=1}^{r} \rho^{j} D_{i}\left(x, y, u_{j}\right),
\end{aligned}
$$

and if the boundary conditions (7.2) hold.

Let $\Omega^{*}$ denote the set of all such generalized solutions $[z, v]$. Let $\Omega^{\prime}$ denote a closed class of generalized admissible pairs, that is $\Omega^{\prime} \subseteq \Omega^{*}$ and whenever $\left[z_{k}, v_{k}\right] \in \Omega^{\prime}, k=1,2, \cdots, z_{k} \rightarrow z$ weakly in $\left(W_{p}^{1}(G)\right)^{n}$, and there is a measurable $v$ such that $[z, v] \in \Omega^{*}$, then there is also (another) measurable function $\bar{v}$ such that $[z, \bar{v}] \in \Omega^{\prime}$.

Since the control variable does not appear explicitly in the definition (2.2) of the functional $I$, it is defined also for generalized solutions, and in this case it is denoted by $I[z, v]$. Let us recall that a generalized optimal pair relative to $\Omega^{\prime}$ is an element $[z, v]$ of $\Omega^{\prime}$ such that $\left.I[z, v] \leqslant I \tilde{z}, \widetilde{v}\right]$ for all $[\tilde{z}, \tilde{v}] \in \Omega^{\prime}$.

Let $V(x, y)=\left\{v(x, y) \mid v=\left(\rho^{1}, \cdots, \rho^{r}, u_{1}, \cdots, u_{r}\right), u_{i}(x, y) \in U(x, y)\right.$, $\rho^{i}(x, y) \geqslant 0, \rho^{i}, u_{i}$ measurable, $\left.i=1, \cdots, r, \Sigma_{i=1}^{r} \rho^{i}(x, y)=1\right\}$ and let $Q(x, y, z)=\left(f_{1}, f_{2}\right)(x, y, z, V(x, y))$ for $(x, y, z) \in G \times E^{n}$. Thus,

$$
\begin{gathered}
Q(x, y, z)=\left\{\left(\zeta_{1}, \zeta_{2}\right) \mid \zeta_{1}=\sum_{j=1}^{n} A_{i j} z^{j}+\sum_{j=1}^{r} \rho^{j} C_{i}\left(x, y, u_{j}\right),\right. \\
\zeta_{2}=\sum_{j=1}^{n} B_{i j} z^{j}+\sum_{j=1}^{r} \rho^{j} D_{i}\left(x, y, u_{j}\right), \\
\sum_{j=1}^{r} \rho^{j}(x, y)=1, u_{j}(x, y) \text { measurable, } \\
\left.u_{j}(x, y) \in U(x, y), j=1, \cdots, r\right\}
\end{gathered}
$$

for $(x, y, z) \in G \times E^{n}$.

By a well-known Carathéodory argument (see Cesari [3]), for $r \geqslant 2 n+1$, the sets $Q(x, y, z)$ are convex. Let us assume that they are also closed. Then, since the differential system (7.4) (and in particular the functions $f_{1}$ and $f_{2}$ ) are linear in $z$, it is seen that the closure theorem (G)(i) of Cesari and Suryanarayana [4] can be applied. [Note that the condition $\left(F_{p}\right)$ of the same paper [4] is satisfied by $\left(f_{1}, f_{2}\right)$.]

Now, we are in a position to prove the following:

THEOREM 3. With the above notation let $G$ be bounded; let $M=$ $\{(x, y, v) \mid v \in V(x, y)\}$ and $Q(x, y, z)$ be closed for $(x, y, z) \in G \times E^{n}$. Let 
$\{z\}_{\Omega^{\prime}}=\left\{z \mid \exists v\right.$ with $\left.(z, v) \in \Omega^{\prime}\right\}$ be weakly compact in $\left(W_{p}^{1}(G)\right)^{n} .\left(\Omega^{\prime}\right.$ is a closed class of generalized admissible pairs.) Then the functional $I[z, v]$ attains its minimum in $\Omega^{\prime}$.

Proof. Let $i=\inf _{\Omega^{\prime}} I[z, v]$. Then, there is a sequence $\left[z_{k}, v_{k}\right] \in \Omega^{\prime}$ such that $I\left[z_{k}, v_{k}\right] \rightarrow i$. Since $\{z\}_{\Omega^{\prime}}$ is weakly compact, there is a subsequence, say still $\left\{z_{k}\right\}$ and an element $z \in\left(W_{p}^{1}(G)\right)^{n}$ such that $z_{k} \rightarrow z$ weakly in $\left(W_{p}^{1}(G)\right)^{n}$. By a known Sobolev theorem (see C. B. Morrey [7]), $z_{k} \rightarrow z$ strongly in $\left(L_{p}(G)\right)^{n}$ and $\left.\left.z_{k}\right|_{\partial G} \rightarrow z\right|_{\partial G}$ strongly in $\left(L_{p}(\partial G)\right)^{n}$. Applying the closure theorem mentioned above, we obtain a measurable function $v(x, y)$ such that $[z, v] \in \Omega^{*}$ and hence by the closedness of $\Omega^{\prime}$, the existence of a measurable function $\bar{v}(x, y)$ with $[z, \bar{v}] \in \Omega^{\prime}$. Furthermore $I\left[z_{k}, v_{k}\right]$ depends only on $z_{k}$ and since "boundary values" of $z_{k}$ converge strongly to those of $z$, it follows that $I[z, v]=i$. This proves the theorem.

REMARK. For a variety of conditions guaranteeing weak compactness of $\{z\}_{\Omega}$ and other details, see Cesari [2a]-[2c], [2e].

8. Existence of usual solutions without convexity. If for every generalized solution of the differential system (7.1), (7.2) there is also a usual solution where the functional $I$ takes the same value, then by Theorem 3, §7, it would follow that usual optimal solutions exist in any closed class of "usual" admissible pairs. The following Lyapunov-type theorem on the range of vector-integrals, is needed in this connection.

THEOREM 4. Let $g^{1}, \cdots, g^{r}$ be fixed elements of $\left(L_{1}(G)\right)^{n}$. Let $Q \subset$ $G$ be a fixed measurable subset. Let $R=\left\{\zeta \in E^{n} \mid \zeta=\sum_{i=1}^{r} \int_{Q_{i}} g^{i} ; Q_{i}, i=1\right.$, $\cdots, r$ disjoint measurable, $\bigcup_{i=1}^{r} Q_{i}=Q$ \} (that is, $R$ is the set of all values of the integral $\Sigma_{i=1}^{r} \int_{Q_{i}} g^{t}$, as $Q_{i}$ describes a decomposition of $Q$ into disjoint measurable subsets).

Let $S=\left\{\zeta \in E^{n} \mid \zeta=\Sigma_{j=1}^{r} \int_{Q}\left(\rho^{j} g^{j}\right)(x, y) d x d y ; \rho^{j}\right.$ measurable, $\rho^{j}(x, y) \geqslant$ $\left.0, j=1, \cdots, r, \Sigma_{j=1}^{r} \rho^{j}(x, y)=1\right\}$. Then $R=S$.

Proof. See Cesari [2f] and [3].

THEOREM 5. Let $G, U(x, y), \varphi_{h i}, A=\left(A_{i j}\right), B=\left(B_{i j}\right), C=\left(C_{i}\right), D=\left(D_{i}\right)$ be as in §2. Let hypotheses (II) and (III) hold (see end of \$4). Then, for every generalized solution $[z, v]$, there is also a usual solution $(w, u)$ with $I[z, v]=$ $I[w, u]$.

Proof. By definition $(z, v)$ satisfies (7.4) which can be written as $z_{x}=A z+P ; z_{y}=B z+Q$ where 


$$
\begin{array}{ll}
P=\left(P_{1}, \cdots, P_{n}\right), & P_{i}=\sum_{j=1}^{r} \rho^{j} C_{i}\left(x, y, u_{j}\right) ; \\
Q=\left(Q_{1}, \cdots, Q_{n}\right), & Q_{i}=\sum_{j=1}^{r} \rho^{j} D_{i}\left(x, y, u_{j}\right) .
\end{array}
$$

Applying the representation (5.3) for $I$, we obtain

$$
I=\nu+\sum_{i=1}^{n} \int_{G}\left(\lambda_{i} P_{i}+\mu_{i} Q_{i}\right) d x d y
$$

where $\nu$ is given by (5.2). Thus,

$$
I=\nu+\sum_{i=1}^{n} \int_{G} \sum_{j=1}^{r} \rho^{j}\left(\lambda_{i} C_{i}\left(x, y, u_{j}\right)+\mu_{i} D_{i}\left(x, y, u_{j}\right)\right) d x d y .
$$

Using Theorem 4 above, it is possible to obtain a decomposition $G_{j}$ of $G, j=$ $1, \cdots, r$, into disjoint measurable subsets $G_{j}$ such that

$$
I=\nu+\sum_{j=1}^{r} \int_{G_{j}} \sum_{i=1}^{n}\left[\lambda_{i} C_{i}\left(x, y, u_{j}\right)+\mu_{i} D_{i}\left(x, y, u_{j}\right)\right] d x d y .
$$

Let $w(x, y), u(x, y),(x, y) \in G$ be the usual solution (admissible pair) defined by

$$
\begin{aligned}
w_{x} & =A w+C(x, y, u) ; \quad w_{y}=B w+D(x, y, u), \quad(x, y) \in G \text { a.e., } \\
w & =\left(w^{1}, \cdots, w^{\eta}\right) ; \quad A=\left(A_{i j}\right) ; B=\left(B_{i j}\right) ; C=\left(C_{i}\right) ; D=\left(D_{i}\right), \\
w^{i}(\sigma) & =\varphi_{h i}(\sigma), \quad \sigma \in s_{h}, i \in\{i\}_{h}, \\
u(x, y) & =u_{j}(x, y), \quad \text { for }(x, y) \in G_{j}, j=1, \cdots, r .
\end{aligned}
$$

Clearly $u(x, y)=u_{j}(x, y) \in U(x, y)$ for $(x, y) \in G_{j}, j=1, \cdots, r$. Since $u$ is measurable, in view of the hypothesis (III) such a pair $(w, u)$ exists. Applying the representation (5.3) once again, for $I(w, u)$ we obtain

$$
\begin{aligned}
I[w, u] & =\nu+\int_{G} \sum_{i=1}^{n} \lambda_{i} C_{i}(x, y, u)+\mu_{i} D_{i}(x, y, u) \\
& =\nu+\sum_{j=1}^{r} \int_{G_{j}} \sum_{i=1}^{n}\left[\lambda_{i} C_{i}\left(x, y, u_{j}\right)+\mu_{i} D_{i}\left(x, y, u_{j}\right)\right] d x d y \\
& =I[z, v] .
\end{aligned}
$$

Hence $[w, u]$ is the required usual solution.

Theorem 6 (Existence of USUAl optimal solutions). Let $G, U(x, y)$, $M, \varphi, A, B, C, D$ be as in $\S 2$ and let hypotheses (II) and (III) hold. Let $G$ be bounded, and $M$ be closed. Let the sets $Q(x, y, z)=\left\{\left(\zeta_{1}, \zeta_{2}\right) \mid \zeta_{1}=A z+\right.$ $\left.C(x, y, u) ; \dot{\zeta}_{2}=B z+D(x, y, u), u \in U(x, y)\right\}$ be closed. Let $\Omega$ be a closed 
class of admissible pairs of usual solutions satisfying the following condition:

(V) The set $\{z(x, y), C(x, y, u(x, y)), D(x, y, u(x, y)):(z, u) \in \Omega\}$ is bounded in $L_{p}$-norm for $p>1$ and bounded by an $L_{1}$-function for $p=1$.

Then, the functional $I[z, u]$ attains its minimum in $\Omega$.

Proof. Under the said hypotheses, the conditions of Theorem 3 are met and hence there exists a generalized optimal solution. Using Theorem 5, there exists also a usual solution where the functional takes the same value. This usual solution is then the required optimal solution in $\Omega$.

REMARK 1. It is to be noted that under condition (V) both sets $\{z\}_{\Omega}$ and $\{z\}_{\Omega^{\prime}}$ are weakly compact in $\left(W_{p}^{1}(G)\right)^{n}$. Here $\{z\}_{\Omega}$ denotes the set of usual trajectories while $\{z\}_{\Omega^{\prime}}$ is the set of generalized trajectories.

REMARK 2. If $G=[a, a+h] \times[b, b+k]$ is a rectangle, then weak compactness of $\{z\}_{\Omega}$ implies that of $\{z\}_{\Omega^{\prime}}$.

Indeed, if $z$ is a generalized trajectory, then there exists controls $u_{j}(x, y)$, $j=1, \cdots, r$, such that,

$$
\begin{gathered}
z_{x}=A z+\sum_{j=1}^{r} \rho_{j} C\left(x, y, u_{j}\right) ; \quad z_{y}=B z+\sum_{j=1}^{r} \rho_{j} D\left(x, y, u_{j}\right), \\
z(x, b)=\varphi_{1}(x) ; \quad z(a, y)=\varphi_{2}(y) .
\end{gathered}
$$

Using assumption (II), we can find usual solutions $z_{j}$ satisfying

$$
\begin{gathered}
z_{j x}=A z_{j}+C\left(x, y, u_{j}\right) ; \quad z_{j y}=B z_{j}+D\left(x, y, u_{j}\right), \\
z_{j}(x, b)=\varphi_{1}(x) ; \quad z_{j}(a, y)=\varphi_{2}(y) .
\end{gathered}
$$

By formal integration and using (8.1), (8.2) we get

$$
\begin{aligned}
z(x, y) & =\varphi_{1}(x)+\exp \left\{\int_{a}^{x} A d \theta\right\} \int_{a}^{x} \sum_{j=1}^{r} \rho_{j} \exp \left\{-\int_{a}^{\xi} A d \theta\right\}\left(z_{j x}-A z_{j}\right) d \xi \\
& =\varphi_{2}(y)+\exp \left\{\int_{b}^{y} B d \theta\right\} \int_{b}^{y} \sum_{j=1}^{r} \rho_{j} \exp \left\{-\int_{b}^{\eta} B d \theta\right\}\left(z_{j y}-B z_{j}\right) d \eta .
\end{aligned}
$$

Now, if $\left\{z_{k}\right\}$ is a sequence of generalized admissible trajectories, let $z_{k j}$ be the corresponding usual trajectories given by (8.2). Since $\{z\}_{\Omega}$ is weakly compact by assumption, there exist $z_{j}^{*} \in\left(W_{p}^{1}(G)\right)^{n}$ and subsequences, say $z_{k j}$ still, $j=$ $1, \cdots, r$, such that $z_{k j} \rightarrow z_{j}^{*}$ weakly in $\left(W_{p}^{1}(G)\right)^{n}$. Let $z^{*}(x, y)$ be defined by (8.3) with $z_{j}$ replaced by $z_{j}^{*}$. Using the linearity of the right-hand side of (8.3), it is seen that $z_{k} \rightarrow z^{*}$ weakly in $\left(W_{p}^{1}(G)\right)^{n}$. This proves weak compactness of $\{z\}_{\Omega}$. 


\section{Bang bang phenomenon.}

Lemma. Let $G \subset E^{2}$ and $U \subset E^{m}$ be two given compact sets. Let $C(x, y, u)=\left(C_{1}, \cdots, C_{n}\right)$ map $G \times U$ into $E^{n}$ and be measurable in $(x, y) \in$ $G$ and continuous in $u \in U$. Let $U^{\prime}=C(x, y, U)=\{C(x, y, u) \mid u \in U\}$. Let $V$ be a closed subset of $U$ such that $\operatorname{co}\left(U^{\prime}\right)=\operatorname{co}\left(V^{\prime}\right)$ where $V^{\prime}=C(x, y, V)$ and co stands for convex hull. Then for each measurable function $u(x, y)$ from $G$ into $U$ there exist measurable functions $\rho_{j}(x, y), u_{j}(x, y), j=1, \cdots$, $n+1$, such that

$$
C(x, y, u(x, y))=\sum_{j=1}^{n+1} \rho_{j}(x, y) C\left(x, y, u_{j}(x, y)\right),
$$

$$
u_{j}(x, y) \in V, \quad \rho_{j}(x, y) \geqslant 0, \quad j=1, \cdots, n+1,
$$

$$
\sum \rho_{j}(x, y)=1,(x, y) \in G
$$

Remarks.1. Since $U$ and $V$ are compact so are $U^{\prime}$ and $V^{\prime}$ as well as $\operatorname{co}\left(U^{\prime}\right)$ and $\operatorname{co}\left(V^{\prime}\right)$.

2. By a theorem of Carathéodory, for $Q \subset E^{n}$, any point of $\operatorname{co}(Q)$ can be written as a convex combination of at most $n+1$ points of $Q$.

Proof of lemma. Given $u: G \rightarrow U, u(x, y) \in U$ and hence $C(x, y, u(x, y)) \in U^{\prime} \subset \operatorname{co}\left(U^{\prime}\right)=\operatorname{co}\left(V^{\prime}\right)$ and hence there are functions $\rho_{j}(x, y)$ and $u_{j}(x, y), j=1, \cdots, n+1$, such that

$$
C(x, y, u(x, y))=\sum_{j=1}^{n+1} \rho_{j}(x, y) C\left(x, y, u_{j}(x, y)\right)
$$

with $\Sigma \rho_{j}=1, \rho_{j} \geqslant 0, u_{j}(x, y) \in V$. It remains to prove that $\rho_{j}$ and $u_{j}$ can be chosen to be measurable. To this end, we use the implicit function theorem (see Proposition 1, §6) as follows: Let $R=\{(x, y, \rho, v) \mid(x, y) \in G, \rho=$ $\left(\rho_{1}, \cdots, \rho_{n+1}\right), v=\left(u_{1}, \cdots, u_{n+1}\right), \rho_{i} \geqslant 0, u_{i} \in V, i=1, \cdots, n+1$, $\Sigma \rho_{i}=1$ \}. Clearly $R$ is a compact subset of $E^{r}$ where $r=3+n+m+n m$. Let $S=\left\{(x, y, z) \mid(x, y) \in G, z \in E^{n}\right\}=G \times E^{n}$. Then $S$ is a closed subset of $E^{2+n}$. Let $F: R \rightarrow S$ be defined by $F(x, y, \rho, v)=\left(x, y, \sum_{i=1}^{n+1} \rho_{i} C\left(x, y, u_{i}\right)\right)$. Clearly $F$ is a continuous function. Let $\theta: G \rightarrow S$ be defined by $\theta(x, y)=$ $(x, y, C(x, y, u(x, y)))$ where $u(x, y)$ is the given measurable function. Since $u(x, y) \in U, \theta(x, y) \in F(R)$ for each $(x, y) \in G$, as observed earlier. Further, $\theta$ is measurable. Hence by the implicit function theorem there is a measurable function $\eta: G \rightarrow R$,

$$
\eta(x, y)=(x, y, \rho(x, y), v(x, y)), \quad v=\left(u_{1}, \cdots, u_{n+1}\right),
$$

such that $\theta=F \eta$, that is,

$$
C(x, y, u(x, y))=\sum \rho_{j}(x, y) C\left(x, y, u_{j}(x, y)\right)
$$


and $\eta$ is measurable implies that $\rho_{j}$ and $u_{j}$ are measurable. Further, $u_{j}(x, y) \in V$.

THEOREM 7. Let $G$ be a Sobolev domain in $E^{2}$ and let $G$ be the union of a countable family of compact subsets. Let $U$ be a fixed compact subset of $E^{m}$. Let $A_{i j}, B_{i j}, C_{i}, D_{i}, P_{h i}, Q_{h i}, i, j=1, \cdots, n$, be as in $\S 2$. (In particular $C$ and $D$ are continuous on $U$.) Let the hypotheses (II) and (III) hold. Let

$$
\begin{aligned}
U^{\prime \prime} & =C(x, y, U) \times D(x, y, U) \\
& \equiv\left\{(\xi, \eta) \in E^{2 n} \mid \xi_{i}=C_{i}(x, y, u), \eta_{i}=D_{i}(x, y, u), u \in U\right\} .
\end{aligned}
$$

Let $V$ be a closed subset of $U$ and let $V^{\prime \prime}$ be defined as $U^{\prime \prime}$ (with $U$ replaced by $V$ that is $V^{\prime \prime}=C(x, y, V) \times D(x, y, V)$. Let $\operatorname{co}\left(U^{\prime \prime}\right)=\operatorname{co}\left(V^{\prime \prime}\right)$.

Then, for every admissible pair $(z, u)$ there is an admissible pair $\left(z^{\prime}, u^{\prime}\right)$ where the functional given by (2.2) takes the same value, and furthermore $u^{\prime}(x, y) \in V$ for almost all $(x, y) \in G$.

Proof. Given the admissible pair $(z, u)$, the functional $I$ can be represented by (5.3) as

$$
\begin{aligned}
I[z, u]=\nu+\sum_{i=1}^{n} \int_{G}\left[\lambda_{i}(x, y) C_{i}(x, y, u(x, y))\right. & \\
& \left.+\mu_{i}(x, y) D_{i}(x, y, u(x, y))\right] d x d y .
\end{aligned}
$$

But, using the above lemma with $C$ replaced by $(C, D)$, there exist measurable functions $\rho_{j}(x, y), u_{j}(x, y), j=1, \cdots, 2 n+1$, such that $\rho_{j}(x, y) \geqslant 0$, $u_{j}(x, y) \in V, j=1, \cdots, 2 n+1, \Sigma \rho_{j}(x, y)=1,(x, y) \in G$ and such that $C(x, y, u(x, y))=\Sigma \rho_{j}(x, y) C_{i}\left(x, y, u_{j}(x, y)\right)$ and similarly for $D$. Thus,

$$
\begin{aligned}
I[z, u]=\nu+\sum_{i=1}^{n} \sum_{j=1}^{2 n+1} \int_{G} \rho_{j}(x, y)\left[\lambda_{i}(x, y) C_{i}\left(x, y, u_{j}(x, y)\right)\right. \\
\left.+\mu_{i}(x, y) D_{i}\left(x, y, u_{j}(x, y)\right)\right] d x d y
\end{aligned}
$$

which by using Theorem 4 can be written as follows in terms of a decomposition $G_{j}$ of $G, j=1, \cdots, 2 n+1$, into disjoint measurable sets.

$$
I[z, u]=\nu+\sum_{i=1}^{n} \sum_{j=1}^{2 n+1} \int_{G_{j}}\left[\lambda_{i} C_{i}\left(x, y, u_{j}\right)+\mu_{i} D_{i}\left(x, y, u_{j}\right)\right] d x d y .
$$

Proceeding similar to proof of Theorem 5, we define $u^{\prime}$ as equal to $u_{j}$ in $G_{f}$ and define $z^{\prime}$ as a corresponding solution of (2.1) with boundary conditions (I). Assumption (III) guarantees the existence of a $z^{\prime}$. Then $\left(z^{\prime}, u^{\prime}\right)$ is admissible and since $\nu$ (see (5.2)) is independent of $u$, we have $I[z, u]=I\left[z^{\prime}, u^{\prime}\right]$. Further $u^{\prime}(x, y) \in V$ for all $(x, y) \in G$. 
REMARKS. 1. If $U$ is a compact set and $V$ is the set of extreme points of $U$, then it is known that $\operatorname{co}(U)=\operatorname{co}(V)$. If $C(x, y, u)=C(x, y) \cdot u$ and $D(x, y, u)=D(x, y) \cdot u$ are linear in $u$, then we also have $\operatorname{co}\left(U^{\prime \prime}\right)=\operatorname{co}\left(V^{\prime \prime}\right)$ and the above theorem applies giving us the existence of optimal bang bang type controls.

2. Following Neustadt [8] one may show that the set $\{I[z, u] \mid(z, u)$ admissible \} "attainability" is closed and convex.

EXAMPLE. (See [9a, p. 239].) Let us consider the problem of the minimum of

$$
I[z, u]=\int_{0}^{a} \int_{0}^{b}\left[A(x, y) z(x, y)+B(x, y) z_{x}(x, y)\right.
$$

$$
\left.+C(x, y) z_{y}(x, y)+f_{0}(x, y, u(x, y))\right] d x d y
$$

with side conditions

$$
\begin{aligned}
z_{x x}+z_{y y}= & D(x, y) z(x, y)+E(x, y) z_{x}(x, y) \\
& +F(x, y) z_{y}(x, y)+f(x, y, u(x, y)),
\end{aligned}
$$

boundary conditions

$$
z(x, 0)=z(x, b)=z(0, y)=z(a, y)=0,
$$

and constraints $u(x, y) \in U \subset E^{m}, U$ fixed and closed. Here, $G$ is the rectangle $[0, a] \times[0, b]$ and the functions $A, B, C, D, E$ and $F$ are assumed to be in $L_{\infty}(G)$.

Introducing the new variables $\Sigma_{1}, \cdots, z_{6}$ given by

$$
\begin{aligned}
z_{1}(x, y) & =\int_{0}^{x} \int_{0}^{y}\left[A z+B z_{x}+C z_{y}+f_{0}(x ; y, u(x, y))\right] d x d y, \\
z_{2} & =z_{1 x} ; \quad z_{3}=z_{1 y} ; \quad z_{4}=z ; \quad z_{5}=z_{x} ; \quad z_{6}=z_{y},
\end{aligned}
$$

the above problem becomes the problem of the minimum of $z_{1}(a, b)=$ $1 / 2 \int_{0}^{a} z_{2}(\alpha, b) d \alpha+1 / 2 \int_{0}^{b} z_{3}(a, \beta) d \beta$ with side conditions

$$
\begin{aligned}
& z_{1 x}=z_{2} ; \quad z_{2 x}=\xi_{1} ; \quad z_{3 x}=A z_{4}+B z_{5}+C z_{6}+f_{0}(x, y, u(x, y)) ; \\
& z_{4 x}=z_{5} ; \quad z_{5 x}=\xi_{2} ; \quad z_{6 x}=\xi_{3} ; \\
& z_{1 y}=z_{3} ; \quad z_{2 y}=A z_{4}+B z_{5}+C z_{6}+f_{0}(x, y, u(x, y)) ; \quad z_{3 y}=\xi_{4} ; \\
& z_{4 y}=z_{6} ; \quad z_{5 y}=\xi_{3} ; \quad z_{6 y}=-\xi_{2}+D z_{4}+E z_{5}+F z_{6}+f(x, y, u(x, y)) ;
\end{aligned}
$$




$$
\begin{aligned}
& z_{1}(x, 0)=z_{2}(x, 0)=z_{4}(x, 0)=z_{5}(x, 0)=0 ; \\
& z_{1}(0, y)=z_{3}(0, y)=z_{4}(0, y)=z_{6}(0, y)=0 ; \\
& z_{4}(x, b)=z_{5}(x, b)=z_{4}(a, y)=z_{6}(a, y)=0 ;
\end{aligned}
$$

and constraints $\left(u, \xi_{1}, \xi_{2}, \xi_{3}, \xi_{4}\right) \in \widetilde{U}=U \times E^{4}$.

The corresponding conjugate problem is given in terms of 12 multipliers $\lambda_{1}, \cdots, \lambda_{6}, \mu_{1}, \cdots, \mu_{6}$. Since $z_{2 x}, z_{6 x}, z_{3 y}$ and $z_{5 y}$ do not appear explicitly in the analysis, we choose $\lambda_{2}=\lambda_{6}=\mu_{3}=\mu_{5}=0$ in $G$. The Hamiltonian in this case is,

$$
\begin{aligned}
H= & \lambda_{1} z_{2}+\mu_{1} z_{3}+\left(\lambda_{3}+\mu_{2}\right)\left(A z_{4}+B z_{5}+C z_{6}+f_{0}\right) \\
& +\lambda_{4} z_{5}+\mu_{4} z_{6}+\lambda_{5} \xi_{2}+\mu_{6}\left(D z_{4}+E z_{5}+F z_{6}+f-\xi_{2}\right)
\end{aligned}
$$

with eight multipliers and the constraints are now $\left(u, \xi_{2}\right) \in U \times E^{1}$. The related conjugate problem is,

$$
\begin{gathered}
\lambda_{1 x}+\mu_{1 y}=0 ; \quad \mu_{2 y}=-\lambda_{1} ; \quad \lambda_{3 x}=-\mu_{1} ; \\
\lambda_{4 x}+\mu_{4 y}=-\left(\lambda_{3}+\mu_{2}\right) A-\mu_{6} \cdot D ; \\
\lambda_{5 x}=-\lambda_{4}-\mu_{6} \cdot E-\left(\lambda_{3}+\mu_{2}\right) \cdot B ; \\
\mu_{6 y}=-\mu_{4}-\mu_{6} \cdot F-\left(\lambda_{3}+\mu_{2}\right) \cdot C ; \\
\lambda_{1}(a, y)=0 ; \quad \lambda_{3}(a, y)=1 ; \quad \lambda_{5}(a, y)=1 ; \mu_{1}(x, b)=0 ; \\
\mu_{2}(x, b)=1 ; \quad \mu_{6}(x, b)=0 ; \quad \lambda_{5}(0, y)=0 ; \mu_{6}(x, 0)=0 .
\end{gathered}
$$

Explicit expressions for one set of solutions of (8.10) are given in [9a] as follows:

Let $\lambda_{1 x}$ be any bounded measurable function on $G$. Let

$$
\begin{array}{ll}
\lambda_{1}(x, y)=\int_{a}^{x} \lambda_{1 x}(\alpha, y) d \alpha ; & \mu_{1}(x, y)=-\int_{b}^{y} \lambda_{1 x}(x, \beta) d \beta ; \\
\lambda_{3}(x, y)=\int_{a}^{x} \int_{b}^{y} \lambda_{1 x}(\alpha, \beta) d \alpha d \beta ; & \mu_{2}(x, y)=\int_{b}^{y} \int_{a}^{x} \lambda_{1 x}(\alpha, \beta) d \alpha d \beta ;
\end{array}
$$$$
\mu_{4}(x, y)=-\left(\int_{0}^{b} \exp \left(\int_{b}^{\beta} F(x, r) d r\right) d \beta\right)^{-1} \int_{0}^{b} 2 C(x, \beta) \exp \left(\int_{b}^{\beta} F(x, r) d r\right) d \beta ;
$$$$
\mu_{6}(x, y)=-\left[\exp \left(-\int_{b}^{y} F\right)\right] \cdot \int_{b}^{y}\left(\mu_{4}+2 c\right)\left(\exp \int_{b}^{\beta} F\right) d \beta ;
$$$$
\lambda_{4}(x, y)=\lambda_{4}(0, y)-\int_{0}^{x}\left(2 A+\mu_{6} D\right)
$$

where 


$$
\begin{aligned}
& \lambda_{4}(0, y)=-a^{-1}\left[1-\int_{0}^{a} d x \int_{0}^{x}\left(2 A+\mu_{6} D\right)+\int_{0}^{a}\left(\mu_{6} E+2 B\right)\right], \\
& \lambda_{5}(x, y)=-\int_{0}^{x}\left(\lambda_{4}+\mu_{6} E+2 B\right) d \alpha .
\end{aligned}
$$

In the above $\mu_{4}(x, y)$ is chosen to be independent of $y$. It may be chosen to depend on $y$ provided the following condition is satisfied:

$$
\int_{0}^{b}\left(\mu_{4}+2 C\right)(x, \beta) \cdot \exp \left(\int_{b}^{\beta} F(x, r) d r\right) d \beta=0 .
$$

The above equation guarantees the boundary conditions on $\mu_{6}$. The above discussion shows that hypothesis (II) of Theorem 6 is satisfied for this problem. Regarding hypothesis (III) on the existence of solutions of the original problem, the literature is extensive and we refer to [1] for a detailed survey. Theorem 6 now gives the existence of optimal solutions in an\% closed class $\Omega$ of admissible pairs satisfying condition (V) of that theorem.

\section{REFERENCES}

1. D. L. Bernstein, Existence theorems in partial differential equations, Ann. of Math. Studies, no. 23, Princeton Univ. Press, Princeton, N. J., 1950. MR 12, 262.

2a. L. Cesari, Existence theorems for multidimensional problems of optimal control, Differential Equations and Dynamical Systems (Proc. Internat. Sympos., Mayaguez, P. R., 1965), Academic Press, New York, 1967, pp. 115-132. MR 36 \#3196.

2b. - Existence theorems for multidimensional Lagrange problems, J. Optimization Theory Appl. 1 (1967), 87-112. MR 37 \#2053.

2c. - Existence theorems for optimal controls of Mayer type, SIAM J. Control 6 (1968), 517-552. MR 39 \#4722.

2d. - Optimization with partial differential equations in Dieudonné-Rashevsky form and conjugate problems, Arch. Rational Mech. Anal. 33 (1969), 339-357. MR 39 \#2040.

2e. - Existence theorems for abstract multidimensional control problems, J. Optimization Theory Appl. 6 (1970), 210-236. MR 42 \#6687.

2f. - Convexity of the range of certain integrals, SIAM J. Control (to appear).

3. - Problems of optimization, Springer-Verlag (to appear).

4. L. Cesari and M. B. Suryanarayana, Closure theorems without seminormality conditions, J. Optimization Theory Appl. (to appear).

5. J. B. Diaz-Lopez, Approximation of generalized solutions by usual solutions in the problems of optimization, Ph. D. Thesis, University of Michigan, Ann Arbor, Mich., 1971.

6. E. J. McShane and R. B. Warfield, On Filippov's implicit function lemma, Proc. Amer. Math. Soc. 18 (1967), 41-47. MR 34 \#8399.

7. C. B. Morrey, Multiple integrals in the calculus of variations, Die Grundlehren der math. Wissenschaften, Band 130, Springer-Verlag, New York, 1966. MR 34 \#2380.

8. L. Neustadt, The existence of optimal control in the absence of convexity conditions, J. Math. Anal. Appl. 7 (1963), 110-117. MR 27 \#4714.

9a. M. B. Suryanarayana, Optimization with hyperbolic partial differential equations, Ph. D. Thesis, University of Michigan, Ann Arbor, Mich., 1969. 
9b. M. B. Suryanarayana, Necessary conditions for optimization problems with hyperbolic partial differential equations, SIAM J. Control 11 (1973), 130-147.

9c. - Existence theorems for optimization problems concerning linear hyperbolic partial differential equations without convexity conditions, J. Optimization Theory Appl. (to appear).

DEPARTMENT OF MATHEMATICS, EASTERN MICHIGAN UNIVERSITY, YPSILANTI, MICHIGAN 48197 